\title{
Comment on "Medication nonadherence in Korean patients with rheumatoid arthritis: the importance of belief about medication and illness perception"
}

Yunjiao Meng and Xujuan Xu

School of Nursing, Nantong University, Nantong, China

Received: April 9, 2018 Accepted: July 18, 2018

\section{Correspondence to}

Xujuan Xu, B.S.

Department of Nursing, Affiliated

Hospital of Nantong University,

2oth Xisi Rd, Chongchuan Qu,

Nantong, 226001, China

Tel: +86-513-85052298

Fax: +86-513-85052080

E-mail: xxj_1124@126.com
I read with great interest the research of Suh et al. [1] published in the Korean Journal of Internal Medicine on January 2018 regarding the status of medication nonadherence in Korean patients with rheumatoid arthritis (RA) and related factors. A total of 292 patients with RA participated in this study. The subjects were divided into two groups, intentional and unintentional, based on the results of a self-reported questionnaire. Each patient completed two iterations of a brief illness perception questionnaire, a belief about medicines questionnaire, and a patient health questionnaire, which provided demographic and clinical data, patient perceptions of illness, treatment beliefs, and mood. Statistical differences of related factors were calculated among different groups; multinomial regression analysis was applied to determine risk factors. The authors also compared the prevalence of unintentional and intentional non-adherence medication adherence, which was stratified by variants of anti-rheumatic medication. The rationale of the study is clear but left me with some thoughts.

Previous studies have rarely used unintentional nonadherence and intentional nonadherence as the criteria for grouping. This distinction is one of the highlights of the paper and it reflects the authors' deeper understanding of medication nonadherence. However, if a patient is both intentional and unintentionally nonadherent, it raises the question how do we accurately classify these behaviors? This is a crucial distinction that I believe needs to be addressed further.

Another pertinent issue of this study is that the questionnaires did not provide verification of the reliability or validity. This lack of methodological vetting raises difficulty as the reader cannot judge whether the questionnaires provide accurate responses, nor can we be certain of the reliability of the results.

Finally, the authors compared the rates of intentional and unintentional nonadherence among different drug types, but there are repetitions in samples between methotrexate group and nonsteroidal anti-inflammatory drugs and/or disease-modifying antirheumatic drugs group. This discrepancy may have affected the results and subsequent conclusions. I would suggest that samples be separated into different group by number [2] or name of drugs such as methotrexate, leflunomide, or 
hydroxychloquine [3].

\section{Conflict of interest}

No potential conflict of interest relevant to this article was reported.

\section{REFERENCES}

1. Suh YS, Cheon YH, Kim HO, et al. Medication nonadherence in Korean patients with rheumatoid arthritis: the importance of belief about medication and illness perception. Korean J Intern Med 2018;33:203-210.

2. Xia Y, Yin R, Fu T, et al. Treatment adherence to disease-modifying antirheumatic drugs in Chinese patients with rheumatoid arthritis. Patient Prefer Adherence 2016;10:735-742.

3. Voshaar M, Vriezekolk J, van Dulmen S, van den Bemt B, van de Laar M. Barriers and facilitators to disease-modifying antirheumatic drug use in patients with inflammatory rheumatic diseases: a qualitative theory-based study. BMC Musculoskelet Disord 2016;17:442. 